\title{
NSBP-1 mediates the effects of cholesterol on insulin/IGF-1 signaling in Caenorhabditis elegans
}

\author{
Mi Cheong Cheong $\cdot$ Hyoung-Joo Lee $\cdot$ \\ Keun Na $\cdot$ Hyoe-Jin Joo $\cdot$ Leon Avery $\cdot$ \\ Young-Jai You $\cdot$ Young-Ki Paik
}

Received: 7 August 2012 / Revised: 30 October 2012 / Accepted: 22 November 2012 / Published online: 20 December 2012

(C) Springer Basel 2012

\begin{abstract}
Nematode sterol-binding protein 1 (NSBP-1) is a homolog of nucleosome assembly protein 1 in mammals that is expressed widely in Caenorhabditis elegans. NSBP-1 mutants are biologically lethal, demonstrating the significance of the gene in growth and development. We investigated how cholesterol influences the insulin signaling pathway through this novel sterol-binding protein in C. elegans. Here we report that NSBP-1 influences many biological processes mediated by insulin signaling, such as longevity, dauer formation, fat storage, and resistance to oxidative stress. We found that NSBP-1 is phosphorylated by AKT- 1 downstream of insulin signaling. In the absence of insulin signaling, NSBP-1 is translocated to the nucleus and binds to DAF-16, a FOXO transcription factor, in a cholesterol-dependent manner. Moreover, NSBP-1 and DAF-16 regulate a common set of genes that can directly modulate
\end{abstract}

Electronic supplementary material The online version of this article (doi:10.1007/s00018-012-1221-0) contains supplementary material, which is available to authorized users.

M. C. Cheong · H.-J. Lee · K. Na · H.-J. Joo · Y.-K. Paik $(\bowtie)$

Department of Biochemistry and Integrated Omics for Biomedical Science (WCU Program), College of Life Science and Biotechnology and Yonsei Proteome Research Center,

Yonsei University, 134 Shinchon-dong, Sudaemoon-ku,

Seoul 120-749, Korea

e-mail: paikyk@yonsei.ac.kr

Present Address:

M. C. Cheong $\cdot$ L. Avery

Department of Physiology and Biophysics,

Virginia Commonwealth University, Richmond, VA 23298, USA

Y.-J. You

Department of Biochemistry and Molecular Biology,

Virginia Commonwealth University, Richmond, VA 23298, USA fat storage, longevity, and resistance to stress. Together, our results present a new steroid-binding molecule that can connect sterol signaling to insulin signaling through direct interaction with FOXO.

Keywords Cholesterol Cholesterol-binding protein . C. elegans · D2096.8 · Insulin/IGF-1 signaling · DAF-16
Abbreviations
FOXO Forkhead transcription factor
HCM High cholesterol medium
LCM Low cholesterol medium
MLS Mean lifespan
NAP Nucleosome assembly protein
NSBP Nematode sterol-binding protein

\section{Introduction}

Cholesterol serves as a plasma membrane component to control membrane fluidity and permeability. It is also a precursor for biologically active molecules, including vitamin D, oxysterols, steroid hormones (such as ecdysone or dafachronic acid), and bile acids. Sterols usually need sterol-binding proteins (SBPs) to regulate sterol homeostasis, trafficking, and cell metabolism. For instance, SREBP and LXR are transcription factors that regulate the expression of genes involved in cholesterol homeostasis [1, 2], lipoproteins and NPCs are transporters of cholesterol, and caveolin located in the membrane regulates both cholesterol transport and signal transduction [3-5]. As in mammals, cholesterol in Caenorhabditis elegans has diverse functions [6-9] including an interaction with the insulin signaling pathway $[10,11]$. A key difference between worms and mammals, however, is 
that worms require only a very small amount of cholesterol, suggesting that cholesterol primarily functions in signaling. For example, it is a precursor of dafachronic acid, a dauer molting hormone.

Insulin is involved in many cellular processes including cell growth and differentiation, carbohydrate metabolism, fat mobilization, lipogenesis, and aging [12-14]. Insulin resistance contributes to the pathogenesis of numerous metabolic diseases, such as type 2 diabetes, hypertension, lipid disorders, atherosclerosis, and reproductive abnormalities $[13,14]$. The insulin pathway has been shown to regulate metabolism, development, stress resistance, and life span is conserved between $C$. elegans and mammals $[15,16]$. In worms, the insulin-like receptor DAF-2 [17] activates a kinase signaling cascade from PI 3-kinase (AGE-1/AAP-1) [18] to downstream serine/threonine kinases such as PDK-1, AKT-1, AKT-2, and SGK-1 [19-21]. Once activated, these kinases negatively regulate the Forkhead (FOXO) transcription factor, DAF-16 [22, 23], which regulates genes involved in longevity, dauer formation, fat accumulation, and stress resistance [22-24]. Recently, FOXO was found to interact with several histone remodeling factors such as the histone deacetylase SIRT1 [25]. This suggests that DAF-16 may differentiate its regulation by inducing different subsets of targets depending on its binding partner.

In this study, we identified nematode sterol-binding protein 1 (NSBP-1), a homolog of nucleosome assembly protein 1 (NAP1) in mammals, as a new cholesterol-binding protein. NSBP-1 interacts with the insulin signaling pathway and is translocated to the nucleus in the absence of the insulin signaling. In the nucleus, it binds to DAF-16 to control metabolic processes such as fat accumulation in a cholesterol concentration-dependent manner. These results suggest that NSBP-1 provides a novel molecular mechanism for DAF-16 to regulate insulin response in conjunction with cholesterol signaling.

\section{Materials and methods}

General methods for culturing worms and strains

All strains were cultured using standard $C$. elegans handling and culture methods [26] with the OP50 Escherichia coli strain as a food source. The wild-type strain was C. elegans variant Bristol, strain N2. Other strains used were CB1370 daf-2(e1370), CF1038 daf-16(mu86), RB759 akt1(ok525), RT130 pwIs23[vit-2::GFP], CF1407 daf-16(mu86) I; muIs71[pKL99 (daf-16a::GFP/bKO) + pRF4(rol-6)]. The daf-2(e1370); muIs84[Psod-3::gfp] strain was generated by crossing daf-2(e1370) males to Psod-3::gfp(muIs84) hermaphrodites.
Expression vector constructs

To produce GST-D2096.8 protein, a D2096.8 cDNA fragment was amplified by PCR and cloned into the EcoRI and NotI sites of pGEX-4T-3. This expression vector was transformed into E. coli Rosetta (DE3), overexpressed, and purified using glutathione-Sepharose $4 \mathrm{~B}$. To produce a GFP expression construct of NSBP-1, a PCR fragment containing the $n s b p-1$ coding region with $1.4 \mathrm{~kb}$ of the upstream promoter region was amplified and cloned into the SphI and BamHI sites of the Fire lab vector pPD 95.75 to generate the NSBP-1::GFP construct. Approximately $50 \mathrm{ng} / \mu 1$ co-injection marker pRF4 (rol-6(sd)) was mixed with the NSBP-1::GFP construct (at a final concentration of $100 \mathrm{ng} / \mu \mathrm{l}$ ). The T203A variant (NSBP-1T203A::GFP) was generated by site-directed mutagenesis of NSBP-1::GFP according to the manufacturer's recommendations (Stratagene, La Jolla, CA, USA). The mutation was verified by nucleotide sequencing. The primers used for PCR analysis are listed in Table S3.

\section{Cholesterol feeding conditions}

Worms were grown in different cholesterol-containing nematode growth medium (NGM) or RNAi plates. Low cholesterol medium (LCM) is the same as NGM, except that no cholesterol was added. High cholesterol medium (HCM) is the same as NGM, except that instead of $5 \mu \mathrm{g} / \mathrm{ml}$ added cholesterol, it contains $20 \mu \mathrm{g} / \mathrm{ml}$. Other conditions were the same as previously described [9].

\section{RNAi assay}

To prepare the bacterial RNAi expression construct, an $n s b p-1$ cDNA fragment was amplified by PCR from C. elegans and cloned into the XbaI and HindIII sites of the Fire lab vector pPD126.36 vector. RNAi plates were prepared by supplementing NGM with $100 \mu \mathrm{g} / \mathrm{ml}$ ampicillin and $1 \mathrm{mM}$ IPTG. RNAi bacteria were grown overnight at $37{ }^{\circ} \mathrm{C}$ in LB media supplemented with $100 \mu \mathrm{g} / \mathrm{ml}$ ampicillin. After 1 day, the cultures were diluted (1:50) into LB broth containing $100 \mu \mathrm{g} / \mathrm{ml}$ ampicillin and grown at $37^{\circ} \mathrm{C}$ to an $\mathrm{OD}_{600}$ of 0.5 , and then $1 \mathrm{mM}$ IPTG was added. After $3 \mathrm{~h}$, approximately $200 \mu \mathrm{l}$ of the bacterial suspension was seeded onto each RNAi plate.

\section{Dauer assay}

For the dauer assays, approximately five adult worms were transferred onto the RNAi bacteria and maintained at $15{ }^{\circ} \mathrm{C}$ for approximately 3 days. $\mathrm{F} 1$ adult worms were then picked, transferred to a fresh RNAi plate, and allowed to lay eggs. Approximately 120 eggs were picked from 
these plates, transferred onto three fresh plates containing the RNAi bacteria, and then incubated at the indicated temperatures. The plates were scored for the presence of dauers or non-dauers after 3.5-4 days, with some exceptions.

Analysis of resistance to oxidative stress

Following NSBP-1 and DAF-16 knockdown, wild-type N2, daf-2, or daf-16 worms were fed E. coli HT115 (DE3) cells expressing the indicated double-stranded RNA. Young adult worms were transferred to an M9 solution containing $100 \mathrm{mM}$ paraquat $\left(1,1^{\prime}\right.$-dimethyl-4,4'-bipyridinium dichloride, Sigma-Aldrich, St. Louis, MO, USA) and incubated at $20^{\circ} \mathrm{C}$. The number of dead worms was counted by the continuous absence of swimming movements and pharyngeal pumping every $5 \mathrm{~h}$ for up to $20 \mathrm{~h}$. Thirty worms from each strain were treated with $100 \mathrm{mM}$ paraquat. This experiment was performed three times.

\section{Lifespan measurement}

Synchronized populations were obtained by allowing adults to lay eggs at $20^{\circ} \mathrm{C}$ for 5-6 h on RNAi plates. After larval development, the worms were transferred onto RNAi plates containing $0.05 \mathrm{mg} / \mathrm{ml} \mathrm{FUdR}$. The number of living animals was scored every 1-2 days until death, which was defined as the failure to respond to gentle prodding on the head and tail with a platinum wire.

Coupling of cholesterol 3-hemisuccinate to EAH Sepharose 4B for cholesterol affinity chromatography

Cholesterol-substituted EAH-Sepharose 4B was prepared according to Cuatrecasas et al. [27-29] with a few modifications. EAH-Sepharose 4B (1 ml, GE Healthcare, Piscataway, NJ, USA) was swollen in $20 \mathrm{ml}$ of $0.5 \mathrm{M} \mathrm{NaCl}$ and washed with $5 \mathrm{ml}$ of distilled water (adjusted to $\mathrm{pH} 4.5$ with $0.1 \mathrm{M} \mathrm{HCl}$ ). The swollen EAH-Sepharose 4B beads were placed in $1 \mathrm{ml}$ of diethylene dioxide (1,4-dioxane) containing $0.0486 \mathrm{~g}$ cholesterol-hemisuccinate $(10 \mathrm{mmol})$ and $1 \mathrm{ml}$ of water ( $\mathrm{pH} \mathrm{4.5)}$. Cholesterol-3 hemisuccinate was coupled to 1,6-diaminohexyl (EAH)-substituted Sepharose $4 \mathrm{~B}$ using the carbodiimide-promoted condensation reaction described previously [30, 31]. Briefly, $0.055 \mathrm{~g}$ of 1-ethyl3(3-dimethylaminopropyl)-carbodiimide $(0.13 \mathrm{M})$ was added

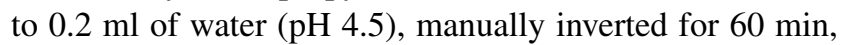
and then stirred for $24 \mathrm{~h}$ at $4{ }^{\circ} \mathrm{C}$. The resulting cholesterolsubstituted EAH-Sepharose 4B was washed successively with $20 \mathrm{ml}$ of 1,4-dioxane, $100 \mathrm{ml}$ of $80 \%$ (v/v) ethyl alcohol, $10 \mathrm{ml}$ of acetone, $20 \mathrm{ml}$ of distilled water, and $20 \mathrm{ml}$ of $10 \mathrm{mM}$ potassium phosphate buffer, $\mathrm{pH} 7.5$ containing $20 \%$ (v/v) glycerol.
Proteomic analysis of NSBP-1 using nano-liquid chromatography-tandem mass spectrometry (LC-MS/MS)

For the identification of purified NSBP-1, nano-LC-MS/MS analysis was performed using an Agilent Nano HPLC 1100 system coupled with a linear trap quadrupole mass spectrometer (Thermo Electron, San Jose, CA) as described [32]. Preparation of capillary columns, mobile phase solution, gradient conditions, and flow rates were the same as described previously [32]. Xcalibur 2.1 (Thermo Electron) was used for peak list generation. Acquisition of mass spectra, scanning of MS/MS, temperature, and collision energy was performed as described [32].

\section{$\left[{ }^{3} \mathrm{H}\right]$-labeled cholesterol-binding assay}

We performed the binding assay as described previously [33]. Each assay tube contained $100 \mathrm{nM}\left[{ }^{3} \mathrm{H}\right]$-labeled cholesterol in buffer A $(50 \mathrm{mM}$ Tris- $\mathrm{HCl}$ at $\mathrm{pH} 7.5$, $150 \mathrm{mM} \mathrm{NaCl}, 1 \mathrm{mM}$ dithiothreitol, $0.1 \%$ Fos-Choline 13, and $0.005 \%$ sodium azide) in a final volume of $100 \mu 1$, and was incubated at $25{ }^{\circ} \mathrm{C}$ in the presence of GST-fused proteins for the indicated times. After incubation, the mixture was washed with $2 \mathrm{ml}$ of buffer $\mathrm{A}$, and the bound protein was eluted with $100 \mu \mathrm{l}$ elution buffer $(10 \mathrm{mM}$ reduced glutathione in $50 \mathrm{mM}$ Tris- $\mathrm{HCl}, \mathrm{pH} 8.0$ ). Eluted fractions were measured for radioactivity using a liquid scintillation counter.

\section{RNA isolation and qRT-PCR}

Total RNA was isolated using the RNAspin Mini isolation kit (GE Healthcare), and reverse transcribed using the Transcriptor First Strand cDNA Synthesis Kit (Roche Diagnostics Corp., Indianapolis, IN, USA). The cDNA was quantified using Nanodrop (ND-1000) and used in qRT-PCR reactions. These reactions were performed on an MJ Research Chromo4 Detector using the QuantiTect SYBR Green PCR kit according to the manufacturer's protocol (QIAGEN, Valencia, CA, USA) using $500 \mathrm{ng}$ of cDNA per sample in a total volume of $50 \mu$. Target genes (i.e., sod-3, $m t l-1$ ) were amplified using specific primers. Amplification and expression analysis were performed in triplicate. mRNA levels in the tested strains were normalized to those in the vector control RNAi group and compared to NSBP-1- or DAF-16-specific RNAitreated samples. Serially diluted vector RNAi cDNA (500-0.05 ng) was used to construct a standard curve for qRT-PCR using actin as an internal control. All of the primers used are listed in Table S3. Unless otherwise specified, the data presented throughout this paper represent the mean $( \pm$ SEM $)$ from three independent experiments performed in triplicate. 
Immunoprecipitation and Western blotting

Wild-type, daf-16::gfp, daf-2(e1370); daf-16::gfp, or Psod-3 $\because g f p$ mixed-staged worms were collected by washing with water, pelleted by centrifugation at $3,000 \times g$ for $30 \mathrm{~s}$, and then frozen in liquid nitrogen in approximately $100-\mu 1$ aliquots. Depending on the size of the frozen worm pellet, an approximately $4 \times$ volume of lysis buffer $(50 \mathrm{mM}$ Tris- $\mathrm{HCl}$ at $\mathrm{pH} 7.5,150 \mathrm{mM} \mathrm{NaCl}, 1 \mathrm{mM}$ dithiothreitol, $0.1 \%$ Fos-Choline 13, $0.005 \%$ sodium azide, and protease inhibitor cocktail) was added. The pellets underwent three freeze/thaws and were then homogenized. For immunoprecipitation, the lysate was incubated with antibody $(10 \mu \mathrm{l}$ antibody per $1 \mathrm{mg}$ total protein) for $2 \mathrm{~h}$ at $4{ }^{\circ} \mathrm{C}$. The antiNSBP-1 antibody was generated against a peptide from the C-terminus of NSBP-1 (FEPPKSKDERNEDED) as reported previously [34]. A 1/10 volume of protein A slurry was then added and the mixture incubated for $4 \mathrm{~h}$ at $4{ }^{\circ} \mathrm{C}$. The protein $\mathrm{A}$ beads were then washed three times with lysis buffer, and bound proteins were eluted by boiling in $4 \mathrm{X}$ sample buffer for $5 \mathrm{~min}$ prior to Western blotting. The primary antibodies used were anti-DAF-16 (rabbit, Santa Cruz Biotechnology, Santa Cruz, CA, USA), anti-GFP (mouse, Santa Cruz Biotechnology), and anti-NSBP-1 (rabbit, Abfrontier, Seoul). Anti-mouse and anti-rabbit secondary antibodies were obtained from Santa Cruz Biotechnology.

Fat assessment

Sudan black staining of stored fat was performed as described previously [17]. Briefly, wild-type and daf-2(e1370) adult worms were transferred onto fresh RNAi plates and grown to the L3 stage. The worms were then washed off the plates and incubated in M9 buffer for $30 \mathrm{~min}$ on a shaker at RT. After three washes with M9 buffer, the worms were fixed in $1 \%$ paraformaldehyde, and then sequentially dehydrated with washes in 25, 50, and $70 \%$ ethanol. Saturated Sudan Black (Sigma-Aldrich) solution was prepared fresh in $70 \%$ ethanol. The fixed worms were incubated for $1-4 \mathrm{~h}$ in $200 \mu \mathrm{l}$ of Sudan Black solution on a shaker at $4{ }^{\circ} \mathrm{C}$.

Oil-Red $\mathrm{O}$ staining was conducted by as previously reported [35]. Briefly, worms collected after RNAi treatment and washed with PBS (pH 7.4) and were then resuspended in $120 \mu \mathrm{l}$ of PBS to which an equal volume of $2 \times$ MRWB buffer containing $2 \%$ paraformaldehyde was added. Samples were gently stirred for $1 \mathrm{~h}$ at room temperature and worms were washed with $1 \times$ PBS. Worms were dehydrated with $60 \%$ isopropanol. Oil-Red-O solution was prepared as follows: a $0.5 \mathrm{~g} / 100 \mathrm{ml}$ isopropanol stock solution equilibrated for several days was freshly diluted to $60 \%$ with water and gently stirred for at least $1 \mathrm{~h}$ and filtrated. The worms were incubated for $1 \mathrm{~h}$ in $1 \mathrm{ml}$ of $60 \%$ Oil-Red O solution at room temperature.
To quantitate total triglyceride content, wild-type and daf-2(e1370) adult worms were picked and transferred onto fresh RNAi plates and grown until the L4 stage. The worms were then frozen in liquid nitrogen and stored at $-80^{\circ} \mathrm{C}$ until needed. Approximately $30 \mathrm{mg}$ of nematodes were weighed and ground in a nitrogen-chilled mortar with $250 \mu$ l of frozen phosphate buffer. Extracts were centrifuged for $7 \mathrm{~min}$ at $12,000 \times g$ to clear the lysate. Fat content was determined using a commercially available TG determination kit (SigmaAldrich) as previously described [36] and normalized to the total amount of protein in each homogenate using the Bradford assay (Bio-Rad, Hercules, CA). For each treatment condition and time point indicated, at least three independent biological samples were generated, with duplicate preparations of lysates from each sample and two measurements for every preparation.

\section{Results}

NSBP-1 binds to cholesterol

In C. elegans, cholesterol is required for reproductive growth and gonad development [7, 8]. Cholesterol functions in part through its binding proteins. However, so far vitellogenin (a functional homolog of mammalian low-density lipoprotein, LDL) is the only known cholesterol-binding protein in worms [37]. To identify new cholesterol-binding protein(s), we used affinity chromatography to screen for cholesterol (sterol)-binding proteins in cell lysates from wild-type worms. We isolated D2096.8 and identified it as a homolog of human NAP1 (with $42 \%$ identity) with proteomic analysis using nano-LC-MS/MS analysis coupled with a linear trap quadrupole mass spectrometer (Thermo Electron, San Jose, CA, USA) as described in the "Materials and methods" section among others including vitellogenin (Online Resource Fig. S1 and Table S1) [37]. We focused on D2096.8 because it was also isolated from our previous screen for daf-2 downstream target genes, yet whether it functions in cholesterol signaling, especially in conjunction with the insulin signaling pathway, had not been addressed $[34,38]$.

To verify that D2096.8 binds to cholesterol, we made a recombinant GST-tagged fusion protein and performed an in vitro cholesterol-binding assay as described previously (Online Resource Fig. S1B) [33, 39]. Recombinant GSTD2096. 8 bound to $\left[{ }^{3} \mathrm{H}\right]$-labeled cholesterol in a concentrationdependent manner with a $K_{\mathrm{d}}$ of $87.5 \pm 27.7 \mathrm{nM}$ (Fig. 1a). The binding was saturated after $30 \mathrm{~min}$ of incubation (Fig. 1b). In competition assays with unlabeled cholesterol, we measured an $\mathrm{IC}_{50}$ of $40 \pm 5.6 \mathrm{nM}$ (Fig. 1c). Competition assays using sterols other than cholesterol (e.g., 7-dehydrocholesterol, desmosterol, $\beta$-sitosterol, 25-hydroxycholesterol, and 
Fig. 1 D2096.8 (NSBP-1)

binds cholesterol.

a GST-D2096.8 binds to

cholesterol in a concentrationdependent manner: D2096.8-

GST (100 pmol) was incubated with increasing concentrations of $\left[{ }^{3} \mathrm{H}\right]$-labeled cholesterol and binding activity measured as described (see "Materials and methods"). b The binding was saturated after $30 \mathrm{~min}$ of incubation: D2096.8-GST was incubated with $\left[{ }^{3} \mathrm{H}\right]$-labeled cholesterol $(100 \mathrm{nM})$ for the indicated time. c D2096.8-GST was incubated with $100 \mathrm{nM}$ $\left[{ }^{3} \mathrm{H}\right]$-labeled cholesterol in the presence of increasing concentrations of unlabeled sterols as indicated. a-c Each data point represents the mean \pm SEM $(n=3)$ from two independent experiments

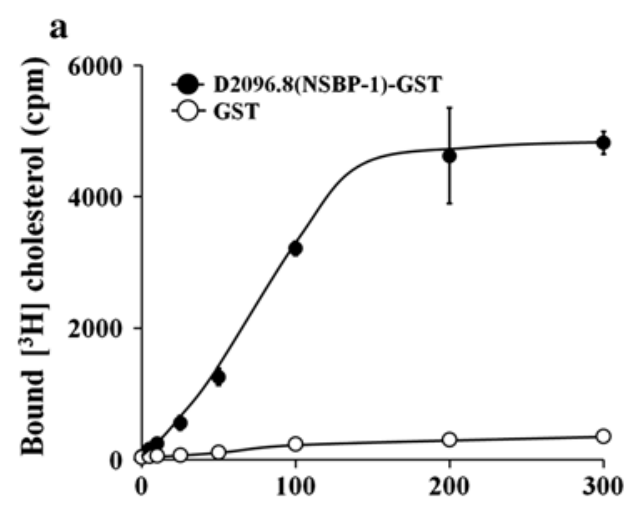

$\left[{ }^{3} \mathrm{H}\right]$ cholesterol concentration(nM)

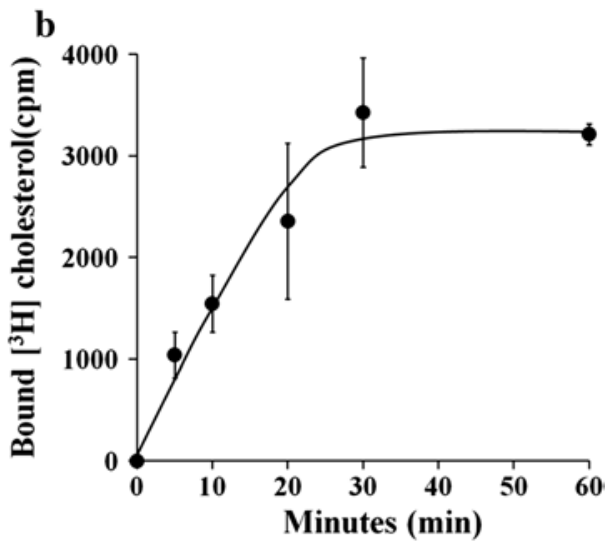

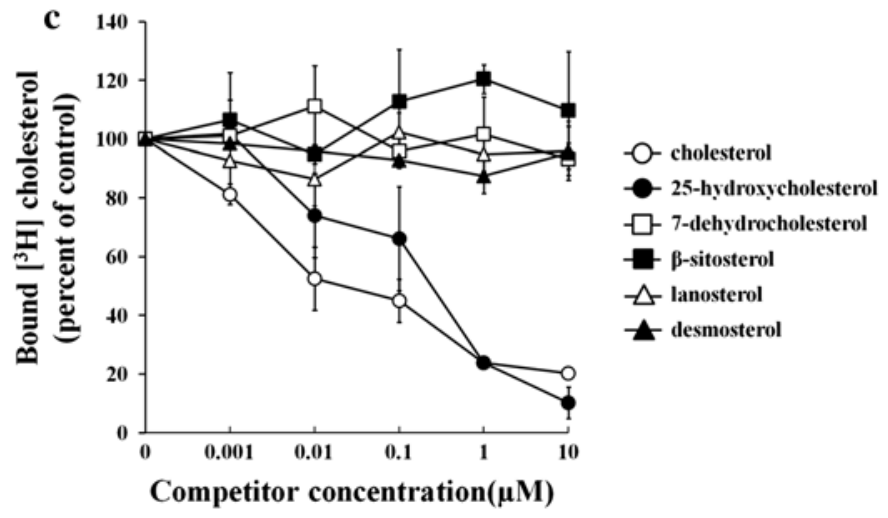

lanosterol) showed that while 25-hydroxycholesterol could compete with $\left[{ }^{3} \mathrm{H}\right]$-labeled cholesterol at a higher concentration ( $\mathrm{IC}_{50} \approx 0.63 \mu \mathrm{M}$ ), no other sterol tested could (Fig. 1c), confirming that D2096.8 binding is mostly specific to cholesterol. We named it nematode sterol-binding protein-1 (NSBP-1).

\section{NSBP-1 function and expression}

To investigate the physiological function of NSBP-1, we examined the phenotype of the $n s b p-1(t m 1278)$ deletion mutant and worms treated with RNAi against $n s b p-1$ (Online Resource Fig S3). nsbp-1 mutants were either arrested as larvae or sterile as adults. Homozygous progeny from a heterozygous mother were scrawny and thin, grew slowly, and showed severe germline defects compared to wild-type siblings (Fig. 2a-e). Even $n s b p-1$ heterozygotes grew noticeably slowly and had reduced brood sizes without any visible defects in either body size or the germline, suggesting haploinsufficiency of this gene in development and reproduction (Online Resource Fig. S2). RNAi knockdown of $n s b p-1$ beginning at the egg stage resulted in viable adults with a normal rate of development. However, a few $n s b p-1(R N A i)$ adults showed abnormal migration of the distal gonad tip, a spermatheca developmental defect, endomitotic oocytes, or abnormal vulval development (Fig. $2 \mathrm{f}-\mathrm{i}$ and data not shown). In contrast, most worms treated with $n s b p-1$ RNAi for two generations showed an abnormal gonad phenotype and became sterile, mimicking the phenotype of $n s b p-1$ mutants. This confirmed that $n s b p-1$ is an essential gene involved in development and growth. These phenotypes mimic the main defects caused by cholesterol deficiency, supporting an NSBP-1 role in cholesterol signaling [7]. To examine where NSBP-1 functions, we generated transgenic worms carrying the $n s b p-1$ promoter and gene fused with GFP to visualize expression. NSBP-1::GFP was widely expressed in various tissues, including the pharynx, vulva, spermatheca, intestine, excretory cells, and hypodermis (Fig. 2j-s), consistent with the pleiotropic phenotypes and broad expression patterns. In the cell, NSBP-1::GFP was expressed diffusely throughout nucleus and cytoplasm.

NSBP-1 influences multiple biological processes affected by the insulin pathway

In the absence of cholesterol, worms arrest as dauer larvae, a dormant form to survive harsh environments $[6,11]$. Dauer formation is regulated by insulin, TGF $\beta$, and cGMP pathways, all of which converge on synthesis of cholesterol-derived ligands of a nuclear hormone receptor, DAF-12 [40]. Because NSBP-1 binds to cholesterol, which regulates the dauer formation pathway, and because 

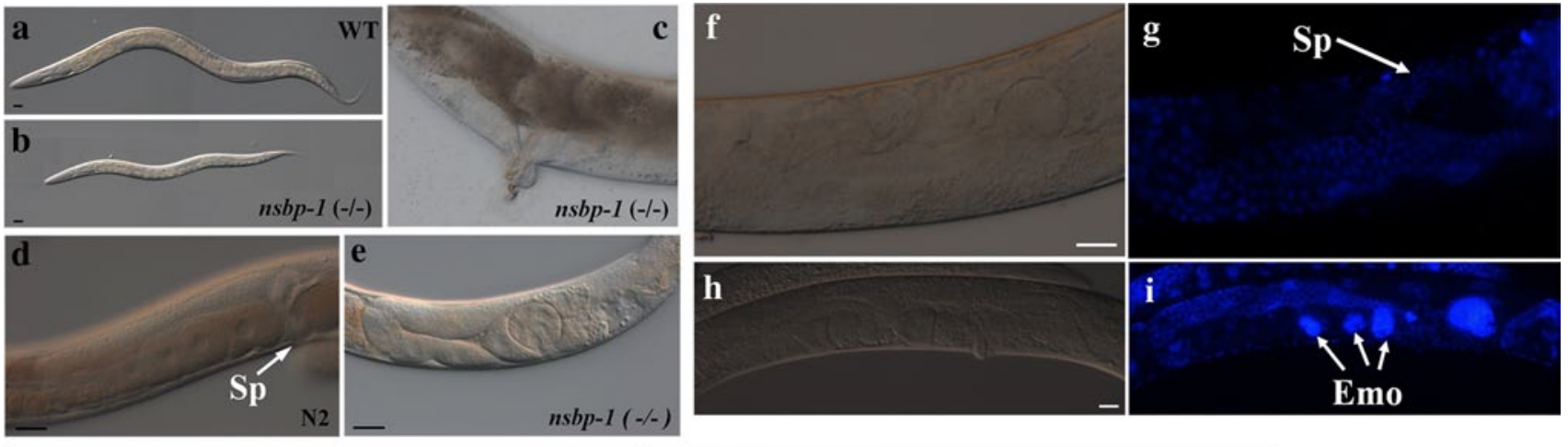
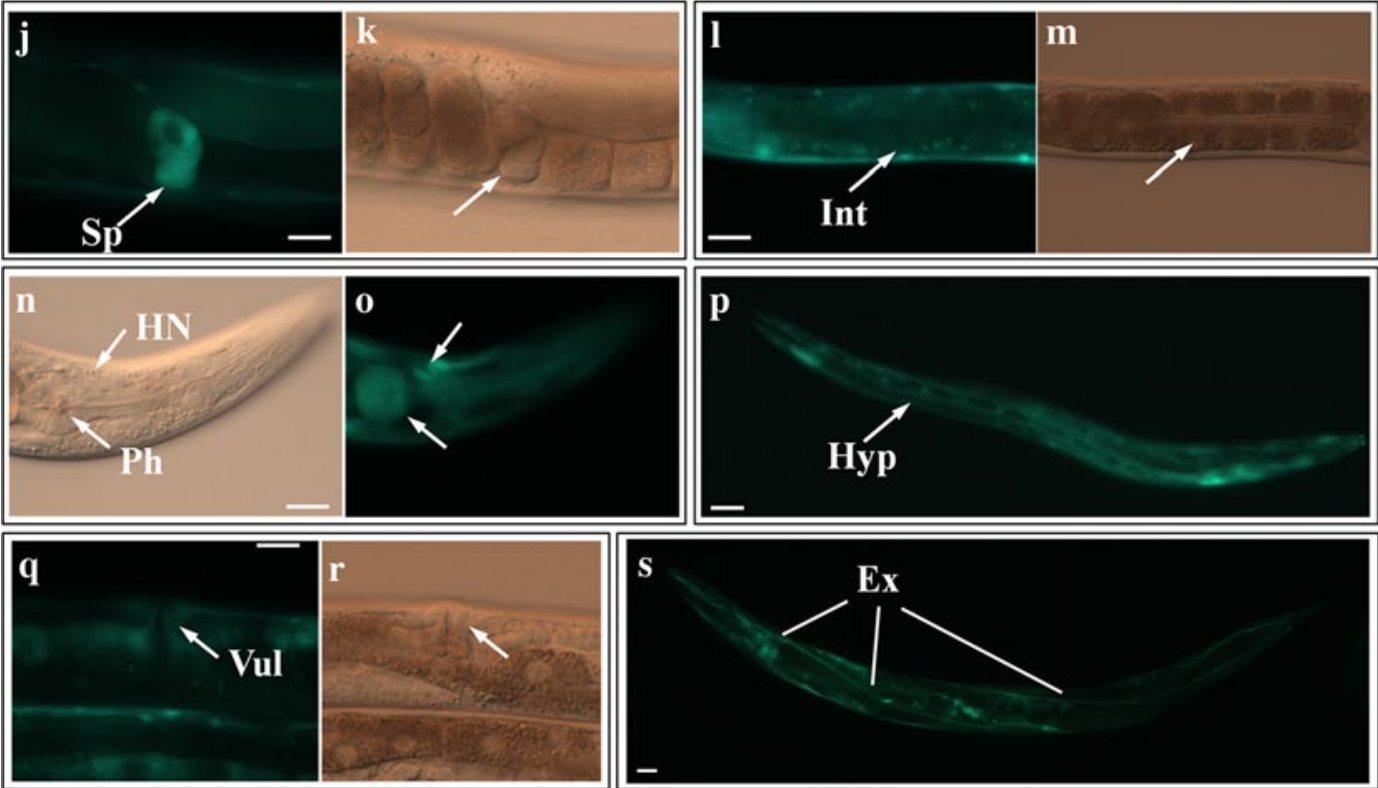

Fig. 2 Phenotypes of the $n s b p-1(t m 1278)$ deletion mutant. a Wild type. Homozygous $n s b p-1$ mutants showed b small body size, c abnormal vulva development, d spermatheca (Sp) of wild type, and e abnormal migration of the distal gonad tip and spermatheca development defects (indicated by arrow). f-i $n s b p-1$ RNAi in the wild type also caused gonad development defects, such as defects in spermatheca development $(\mathbf{f}, \mathbf{g})$ and endomitotic oocytes $(\mathbf{h}, \mathbf{i})$. $\mathbf{g}$ and $\mathbf{i}$ were stained by DAPI and (f) and (h) are corresponding DIC images (Emo endomitotic oocyte, $S p$ spermatheca). $\mathbf{j}-\mathbf{s}$ Transgenic worms

NSBP-1 is located at the downstream of DAF-2 signaling [34], we tested if NSBP-1 regulates dauer formation in daf2 mutants. Since $n s b p-l$ is an essential gene, we used RNAi to partially reduce gene activity. When fed control RNAi, daf-2 mutants became dauers at $25{ }^{\circ} \mathrm{C}$. However, knockdown of $n s b p-1$ in $d a f-2$ mutants reduced dauer formation (Fig. 3a), suggesting NSBP-1 interacts with the insulin signaling pathway.

We further examined life span, fat storage, and resistance to oxidative stress, all of which are known to be regulated by the insulin signaling pathway [41]. RNAi of $n s b p-1$ reduced the lifespan of daf-2 mutant by approximately $33 \%$ compared to control RNAi (Fig. 3b). To carrying NSBP-1::GFP in a wild-type background showed expression of the protein in the spermatheca $(\mathrm{Sp}, \mathbf{j}, \mathbf{k})$, the intestine (Int, $\mathbf{l}, \mathbf{m})$, head neurons and pharynx ( $\mathrm{HN}$ and $\mathrm{Ph}, \mathbf{n}, \mathbf{o})$, the hypodermis (Hyp, $\mathbf{p})$, the vulva (Vul, q, r) and the excretory cell (Ex, s). A representative image from one of three independent experiments is shown $(n=30)$. $\mathbf{j}-\mathbf{s}$ Fluorescence images of transgenic worms while $\mathbf{k}, \mathbf{m}$, $\mathbf{o}, \mathbf{r}$ show the DIC images corresponding to $\mathbf{j}, \mathbf{l}, \mathbf{n}, \mathbf{q}$. All scale bars represent $20 \mu \mathrm{m}$

examine resistance to oxidative stress, as a measure of increased sod-3 expression, we measured the survival of worms treated with $100 \mathrm{mM}$ paraquat. After $20 \mathrm{~h}$ of paraquat treatment, $90 \%$ of control RNAi-treated daf-2 mutants survived, whereas only $62 \%$ of $n s b p-1$ RNAi-treated daf-2 mutants did (Fig. 3c). Lastly, RNAi of $n s b p-1$ reduced fat storage in the daf-2 mutant as measured by Sudan black staining [17] (Fig. 3d). These data show that $n s b p-1$ may have a partial role in mediating dauer formation, longevity, oxidative stress resistance, and fat accumulation in daf-2 mutants, suggesting that $n s b p-l$ influences multiple biological processes regulated by the insulin signaling pathway in C. elegans. 

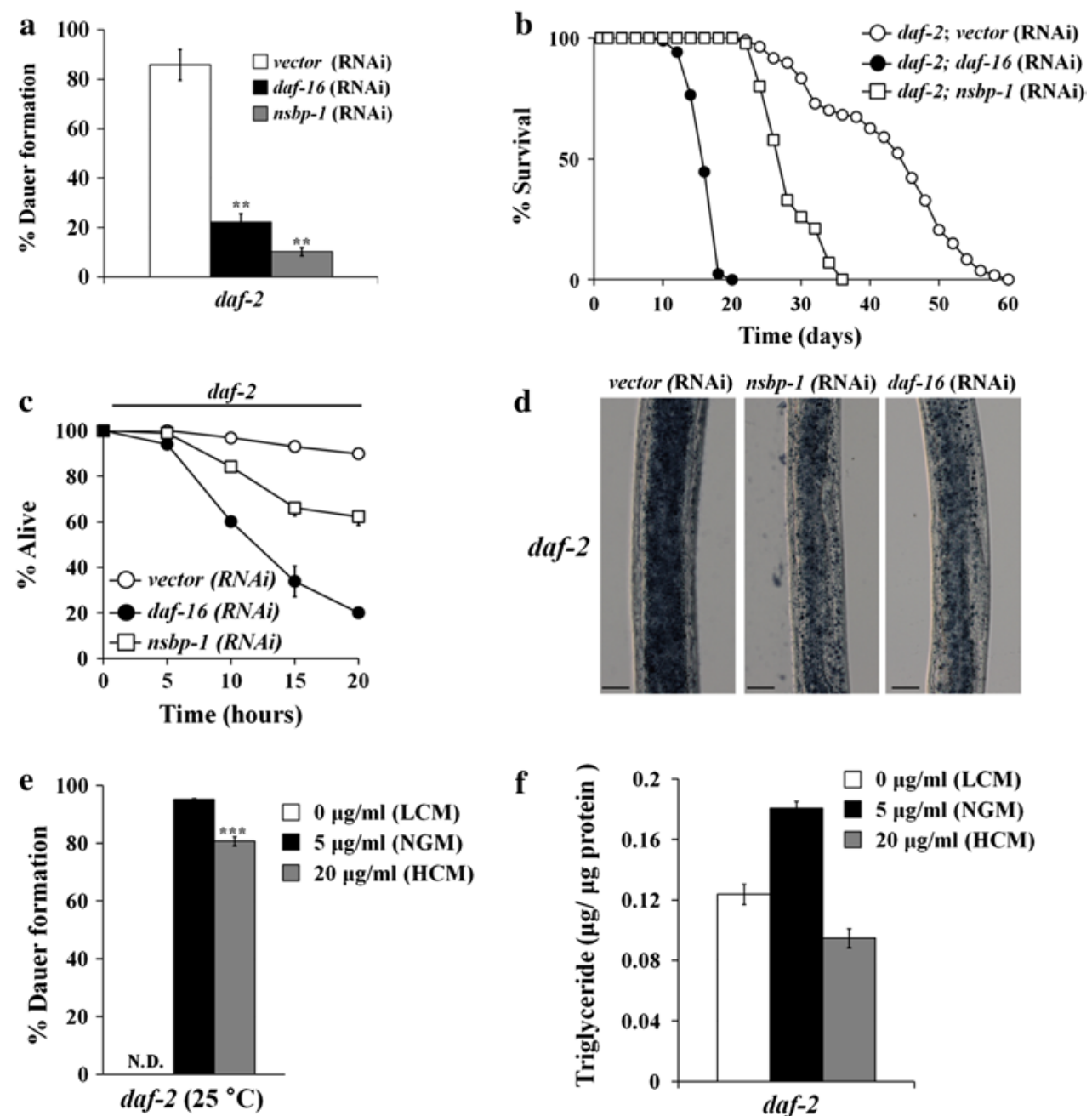

Fig. 3 NSBP-1 influences multiple biological processes affected by the insulin pathway. a $n s b p-1$ knockdown decreased dauer formation in daf-2 mutants at $25{ }^{\circ} \mathrm{C}$. ${ }^{* *} p \leq 0.001$. Unless described otherwise, all values represent the average of three independent experiments $(n \geq 100)$ and error bars indicate the SEM. $p$ values were calculated using Student's $t$ test. b $n s b p-1$ knockdown reduced the lifespan of daf-2 mutants. Mean lifespan [days \pm SEM (sample number)] of control RNAi $=42.7 \pm 0.5$ days $(n=100), n s b p-1$ RNAi $=28.4 \pm 0.4$ days $(n=90), p<0.0001$; daf-16 RNAi $=16.3 \pm 0.2$ days $(n=85), p<0.0001$. c $n s b p-1$ knockdown reduced resistance to oxidative stress in daf-2 mutants. Percent living animals at $20 \mathrm{~h}$ were $90 \%$ (vector RNAi), $20 \%$

Because NSBP-1 binds to cholesterol and because knockdown of $n s b p-1$ by RNAi alleviates several $d a f-2$ mutant phenotypes, we reasoned that if we manipulated cholesterol concentrations to disrupt $n s b p-1$ function, daf-2 might show phenotypes similar to those caused by treatment with $n s b p-1$ RNAi. When we treated daf-2 mutants with three different cholesterol concentrations, $0 \mu \mathrm{g} / \mathrm{ml}$ (low cholesterol medium, LCM), $5 \mu \mathrm{g} / \mathrm{ml}$ (NGM) and $20 \mu \mathrm{g} / \mathrm{ml}$ (high cholesterol medium, HCM), we found that not only low cholesterol but also high cholesterol treatment reduced

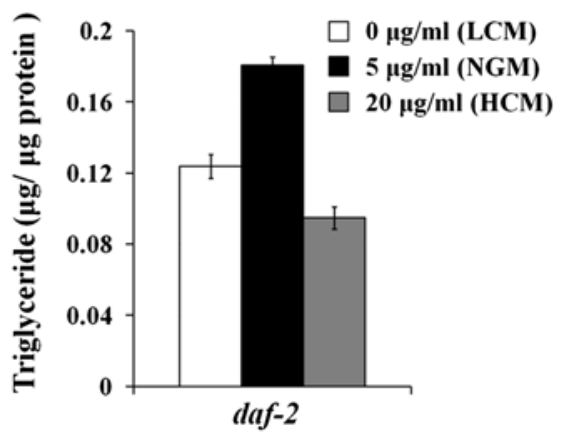

(daf-16 RNAi, $p<0.001$ ), and $62 \%$ (nsbp-1 RNAi, $p<0.001$ ). Worms ( $n=30$ for each strain) were treated with paraquat and then survival was assessed. $p<0.001$ at the 20 -h time point. d $n s b p-1$ knockdown reduced fat accumulation in $d a f-2$ mutants similarly to daf-16 knockdown. A representative image from one of three independent experiments $(n=20)$ is shown. e Cholesterol imbalance decreased dauer formation in daf-2 mutants $25^{\circ} \mathrm{C}(n \geq 100) . N D$ not detected. ${ }^{* * *} p \leq 0.001$ ( $L C M$ low cholesterol medium; $N G M$ normal cholesterol medium; HCM high cholesterol medium; see key in panel f). f Cholesterol imbalance reduced fat storage in daf-2 worms. Total triglyceride content was altered in daf-2 worms in response to changes in cholesterol concentration

dauer formation and fat storage, mimicking the nsbp-l RNAi effect on daf-2 mutants (Fig. 3e, f). That cholesterol imbalance induced a similar phenotype as treating $d a f-2$ mutants with $n s b p-1$ RNAi suggests that cholesterol is necessary for $d a f-2$ mutants to show their phenotypes and the cholesterol function is mediated by $n s b p-1$. However, it is not clear why increased and decreased cholesterol reduces dauer formation and fat storage in daf- 2 animals. We speculate that there may be another factor that can sense the level of cholesterol (e.g., SCAP in mammals) and mediate 

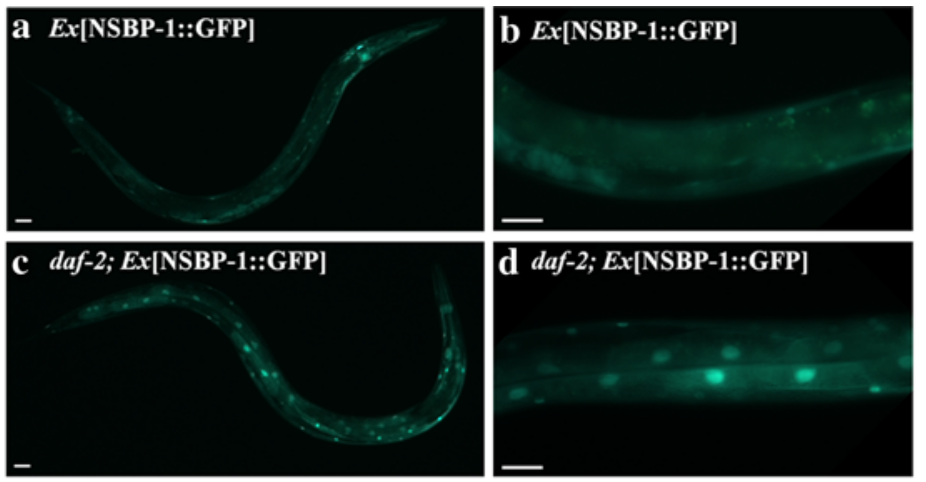

Wild type
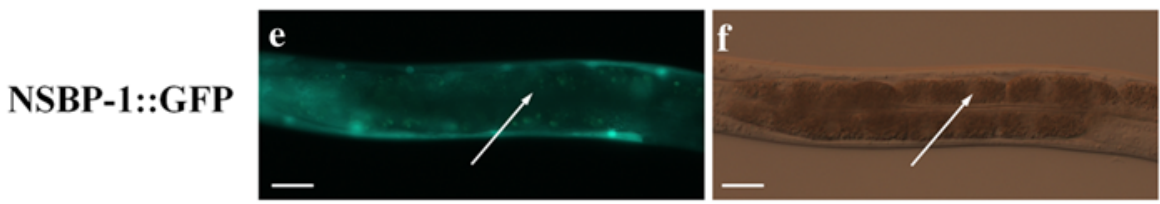

NSBP-1::GFP T203A
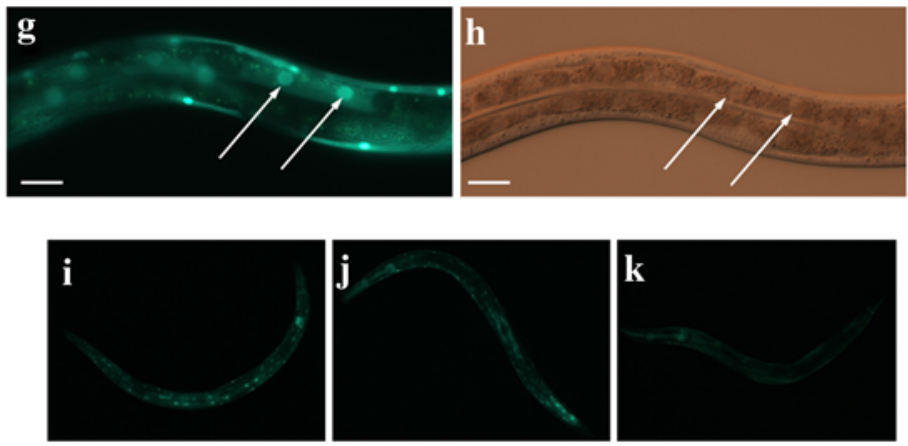

\begin{tabular}{|c|c|c|c|c|}
\hline Strain & High (\%) & Medium (\%) & Low (\%) & (n) \\
\hline$E x[N S B P-1:: G F P]$ & $0.6 \pm 0.6$ & $16.6 \pm 1.6$ & $82.7 \pm 2.2$ & $30,28,31$ \\
\hline daf-2; Ex[NSBP-1::GFP] & $93.3 \pm 1.9$ & $6.7 \pm 1.9$ & 0 & $30,30,31$ \\
\hline$E x[$ NSBP-1::GFP T203A] & $58.9 \pm 4.9$ & $27.8 \pm 2.9$ & $13.3 \pm 1.9$ & $30,28,29$ \\
\hline
\end{tabular}

Fig. 4 NSBP-1 translocated into the nucleus in the absence of insulin signaling. a-d NSBP-1::GFP accumulated in intestinal nuclei when DAF- 2 activity was reduced. a, b NSBP-1::GFP in the wild type. c, d NSBP-1::GFP in daf-2 mutants. e-h Alanine substitution of a predicted AKT phosphorylation site within NSBP-1 (T203A) resulted in nuclear accumulation of NSBP-1::GFP. $E$, NSBP-1::GFP in the wild type (f is its DIC image), $g$ NSBP-1T203A::GFP in the

concentration-dependent modulation of cholesterol effect on such daf-2 mutant phenotypes.

NSBP-1 is translocated into the nucleus in the absence of insulin signaling

To understand the molecular mechanisms by which $n s b p-1$ regulates the insulin signaling pathway, we first compared the localization of NSBP-1 in the wild type to that in daf2 mutants. In wild-type intestinal cells NSBP-1 protein was diffusely localized throughout the cell. However, in wild type (h is its DIC image). White arrows indicate the intestinal nuclei. $\mathbf{a}-\mathbf{h}$ A representative image from one of three independent experiments is shown $(n=30)$. $\mathbf{i}-\mathbf{k}$ Quantitation of NSBP-1::GFP and NSBP-1T203A::GFP in intestinal nuclei; $\mathbf{i}$ indicates high, $\mathbf{j}$ indicates medium, and $\mathbf{k}$ indicates low level of NSBP-1::GFP in intestinal nuclei

daf-2 mutants, NSBP-1 protein was mainly in the nucleus, showing that in the absence of insulin signaling, NSBP-1 proteins translocated into the nucleus (Fig. 4a-d, i-k). Because DAF-16, the main downstream target of the insulin signaling pathway, also translocates into the nucleus in the absence of insulin signaling $[42,43]$ and because it is known that NAP-1 family proteins, homologs of NSBP-1, also translocate into the nucleus to function [44], we hypothesized that NSBP-1 might change its intracellular localization through phosphorylation by kinases in the insulin pathway. 
AGE-1, a worm homolog of PI3 kinase, and AKT-1 are two kinases downstream of daf-2. Mutants for these genes show phenotypes identical to those of daf-2 mutants; they become dauers because the mutants cannot phosphorylate DAF-16 to keep it in the cytoplasm. As a result, DAF-16 gets dephosphorylated and then translocates into the nucleus. In age- 1 and akt- 1 mutants, NSBP-1 was localized in the nuclei (Online Resource Fig. S4, S5), showing that the cytoplasmic localization of NSBP-1 was dependent on insulin signaling pathway kinases. Interestingly, however, NSBP-1 was still localized in the nucleus in daf-16; daf-2 double mutants (Online Resource Fig. S4). This result was verified by treating daf-16 and wild-type worms with daf-2 RNAi; daf-2 RNAi translocates NSBP-1 from cytosol to nucleus in both wild-type and daf-16 worms (Online Resource Fig. S5, S6). These results indicated that NSBP-1 translocates into the nucleus when insulin signaling is absent. However, the translocation does not seem to require DAF-16.

To determine whether phosphorylation plays a role in NSBP-1 localization in the absence of insulin signaling, we searched for potential AGE-1 or AKT-1 phosphorylation sites using the SCANSITE program (http://scansite.m it.edu/). This analysis identified threonine 203 (Thr203) and serine 239 (Ser239) of NSBP-1 as potential AKT-1 phosphorylation sites. To test if either of these was the phosphorylation site to regulate NSBP-1's localization in the absence of insulin signaling, we generated three different transgenic worms that carried NSBP-1 fused with GFP, [36] a construct in which Thr203 of NSBP-1::GFP was substituted with alanine, or [34] a construct in which Ser239 of NSBP-1::GFP was substituted with glycine [45]. NSBP-1 T203A::GFP was constitutively localized in nuclei even in the wild type, whereas NSBP-1 S239G::GFP and NSBP-1::GFP remained in the cytoplasm (Fig. 4e-h, i-k and Online Resource Fig. S7). These results strongly suggest that phosphorylation of NSBP-1 on threonine 203, most likely by AKT-1, keeps NSBP-1 proteins in cytoplasm in the presence of insulin signaling, and dephosphorylation of the site in the absence of insulin signaling translocates the protein into the nucleus.

\section{NSBP-1 directly binds to DAF-16}

Our results show that NSBP-1 can be located in nuclei, and that translocation was dependent on the insulin signaling pathway. Based on the similarity between NSBP-1 and DAF-16, which also translocates into nucleus in the absence of insulin signaling, we examined if NSBP-1 directly interacts with DAF-16 using co-immunoprecipitation (Fig. 5). We used daf-16; muIs 71 worms, which express GFP fused to DAF-16 [42], because it is difficult to detect endogenous DAF-16 in wild-type worms. DAF-16::GFP was detected in daf-16; muIs 71 but not in the wild type or daf-2 (Fig. 5a). To confirm that the interaction was specific to DAF-16 but not to GFP, we immunoprecipitated NSBP-1 from a lysate

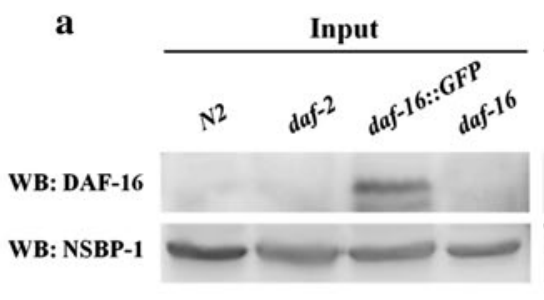

c

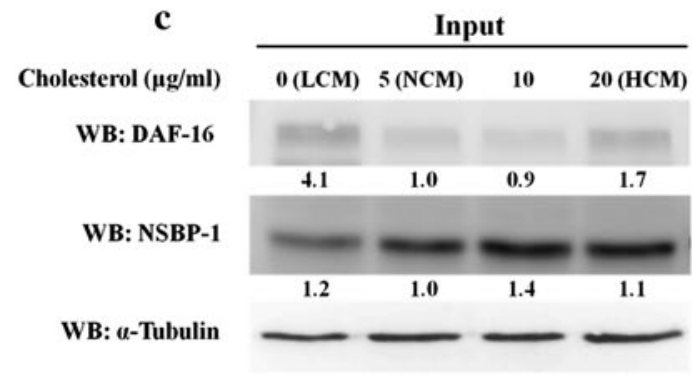

IP: NSBP-1

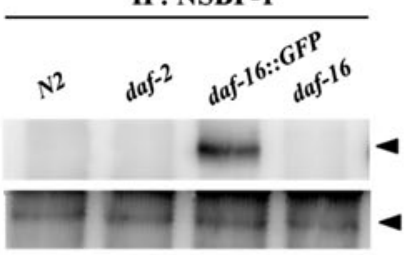

b

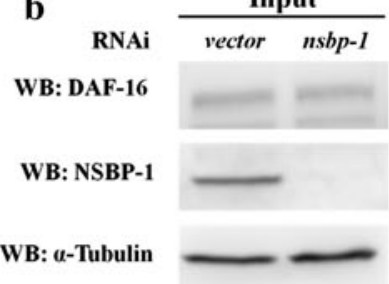

IP: NSBP-1

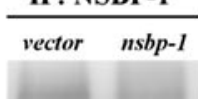

Fig. 5 NSBP-1 directly binds to DAF-16. a Lysates from the indicated genotypes were co-immunoprecipitated (IP) with antibodies against NSBP-1, then analyzed by Western blot (WB) with antiDAF-16 and anti-NSBP-1 antibodies. b Transgenic worms carrying daf-16::GFP were grown on RNAi plates containing L4440 vector or $n s b p-1$ RNAi bacteria. Lysates were co-immunoprecipitated using anti-NSBP-1 antibody and analyzed by Western blot with
IP: NSBP-1

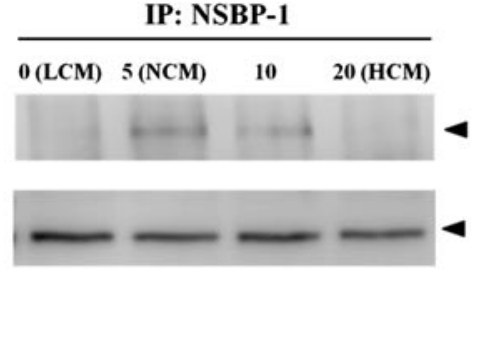

anti-DAF-16 and anti-NSBP-1 antibodies. c NSBP-1 and DAF-16 interaction was affected by cholesterol concentration. Transgenic worms carrying daf-16::GFP were grown at various cholesterol concentrations. Lysates were co-immunoprecipitated using anti-NSBP-1 antibody and analyzed by Western blot with anti-DAF-16 and antiNSBP-1 antibodies. Quantification of fold change in protein normalized with $\alpha$-tubulin is shown below each lane 
muIs84[Psod-3::GFP] worms, which express an unrelated GFP fusion protein. There was no detectable interaction (Online Resource Fig. S8), confirming that the interaction is specific for DAF-16 but not for GFP. To further confirm the specificity, we performed RNAi of $n s b p-l$ to reduce amounts of NSBP-1 protein. No DAF-16 could be immunoprecipitated with NSBP-1 antibody when $n s b p-1$ was knocked down by RNAi, confirming the specificity (Fig. 5b).

Because NSBP-1 also binds to cholesterol, we examined whether binding between NSBP-1 and DAF-16 is regulated by cholesterol. When we examined their interaction using cell lysates from wild-type worms grown in various cholesterol concentrations, both extracts obtained from worms grown in low and high (but not normal cholesterol) medium show reduced in binding between NSBP-1 and DAF-16 (Fig. 5c). This suggests the binding prefers an optimum concentration of $\sim 5 \mu \mathrm{g} / \mathrm{ml}$ cholesterol. This also supports our previous observation (Fig. 3) of a cholesterol imbalance effect on daf-2 mutants; both high and low cholesterol treatment alleviated some of $d a f-2$ mutant phenotypes (fat accumulation and dauer phenotype). This suggests that when there was too much or too little cholesterol, NSBP-1 could not bind to DAF-16 and therefore DAF-16 activity was reduced, which in turn alleviated several daf-2 phenotypes in life span, fat storage, and oxidative stress.

\section{NSBP-1 and DAF-16 co-regulate transcription response}

We have shown that both NSBP-1 and DAF-16 are regulated by the insulin signaling pathway and both translocate into the nucleus in the absence of insulin signaling. Furthermore, we showed that NSBP-1 and DAF-16 bind to each other. To understand the physiological consequences of this binding, we measured gene expression of known daf-16 targets, sod-3 and $m t l-1$, using quantitative RT-PCR (qRT-PCR). The expression of both genes decreased by $n s b p-1$ RNAi (Fig. 6a), indicating that NSBP-1 regulates DAF-16 transcriptional activity positively.

Fatty acid and cholesterol metabolism are related in many aspects of lipid metabolism [46]. Of all the insulin-mediated physiological processes that NSBP-1 influenced, fat storage was most affected (Fig. 3). To understand how nsbp-1 regulates fat accumulation in interaction with DAF-16, we examined four genes that are known to function most significantly in fat accumulation and storage, fat-7, sbp-1, lipl-4, and $\mathrm{C} 46 \mathrm{C} 11.1$. Among them we found that $s b p-1$, a homolog of SREBP, whose expression is known to be regulated by FOXO in mammals [47], is regulated by both NSBP-1 and DAF-16; when we measured $s b p-1$ mRNA level in worms treated with $n s b p-1$ - or $d a f-16$-specific RNAi, $s b p$ - 1 expression was decreased (Fig. 6a). These results indicated that NSBP-1 and DAF-16 act together to regulate both oxidative stress and fat storage.
Increased expression of NSBP-1 enhanced DAF-16 activity

To investigate if $n s b p-1$ overexpression (nsbp- $1 O / E$ ) influences insulin signaling pathway and daf-16 activity, we examined the effects of $n s b p-1 O / E$ on life span, stress resistance, fat storage, and sod-3 expression. Lifespan increased about $25 \%$ in both $n s b p-1(O / E)$ and $n s b p-1(T 203 A O / E)$ worms (Fig. 6b, Online Resource Table S3). In addition, $n s b p-1(O / E)$ worms are resistant to oxidative stress when changes in survival rate in parquet treatment and in sod-3 expression were measured as an index of oxidative stress; after $20 \mathrm{~h}$ of paraquat treatment, nsbp-1(O/E) worms showed $78 \%$ survival, whereas control worms exhibited $60 \%$ viability (Fig. 6c). Fat storage and sod-3 expression were also increased in both $n s b p-1(O / E)$ and $n s b p-1(T 203 A$ $O / E$ ). However, increase in fat storage by overexpression of wild-type or constitutively active NSBP-1 was not abolished by the daf-16 mutation, suggesting that NSBP-1 may have another way to regulate fat storage independent of DAF-16 (Fig. 6d-h). The increases in resistance to oxidative stress, in sod-3 expression and in lifespan were all abolished in daf-16; nsbp-1(O/E) (Fig. 6i). That the phenotypes caused by overexpression and constitutively active gene function show phenotypes exactly opposite to those caused by knockdown of the gene shows that all phenotypes are indeed mediated by $n s b p-1$. Moreover, that the phenotypes caused by overexpression mimic daf-2 mutant phenotypes mediated by DAF-16 and that most of those phenotypes were abolished by DAF-16 mutation strongly supports the hypothesis that NSBP-1 interacts with the insulin pathway by regulating DAF-16 activity.

\section{Discussion}

Although C. elegans lack de novo cholesterol biosynthesis, they require cholesterol for germ cell development, growth, stress resistance, dauer formation, fat storage, cuticle formation, and modulation of other lipids [7, 9, 10, 48-50]. Changes in exogenous or endogenous cholesterol profoundly affect $C$. elegans cellular function and cholesterol cannot be replaced by other lipid molecules. For example, disturbances in cholesterol uptake are known to cause defects in germ cell development, growth, cuticle development, and motility [48, 51]. In this regard, C. elegans represents a nice model for studying cholesterol-mediated signaling [9]. Thus, we believe that many of the physiological changes caused by various cholesterol conditions (e.g., disturbance in uptake or hypercholesterolemia) are relevant to worm physiology [9].

It will be intriguing in the future to determine whether the link between cholesterol and insulin signaling via NSBP-1 that we discovered in C. elegans can also exist in a 

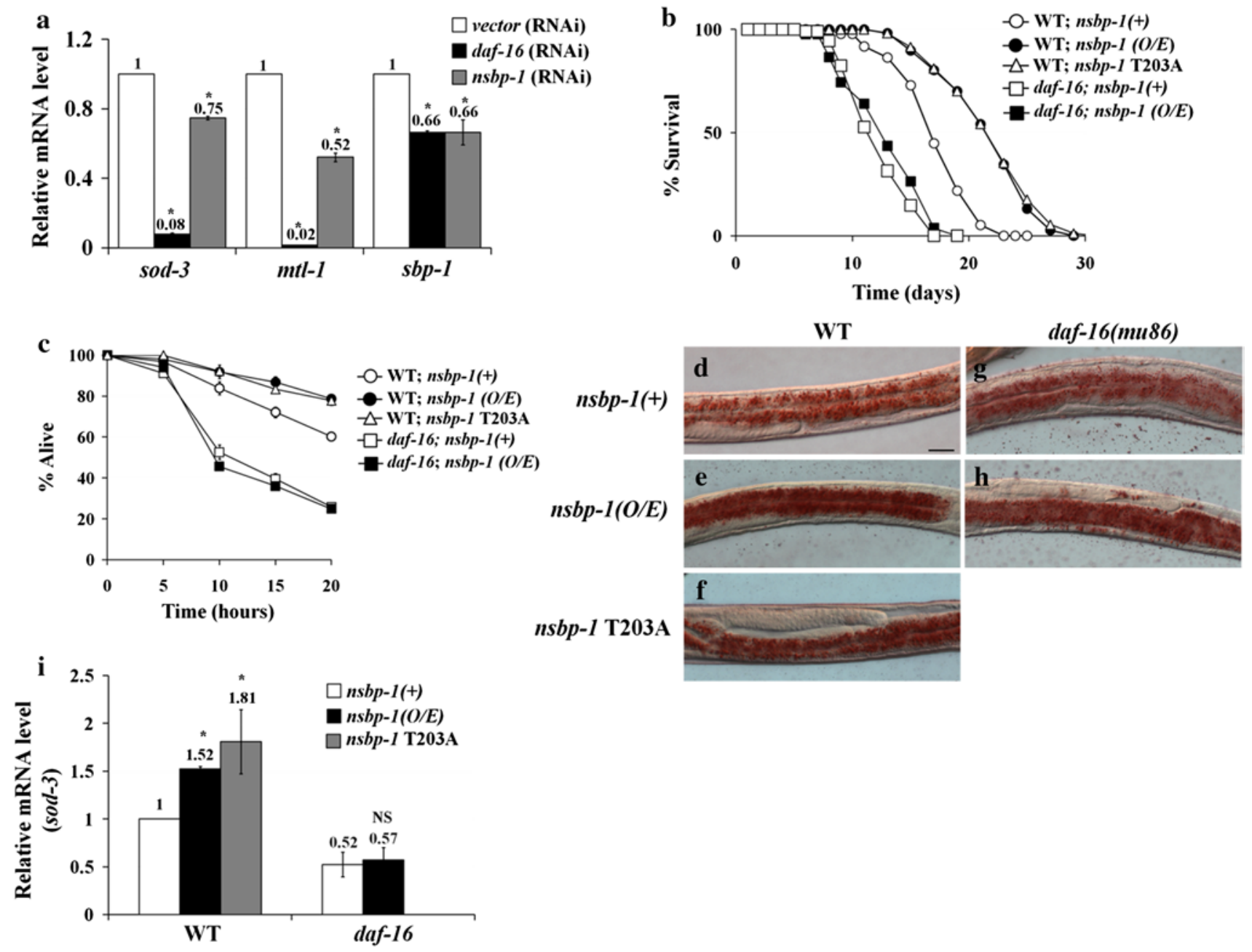

Fig. 6 NSBP-1 regulates DAF-16-dependent transcription. a Decreased sod-3, $m t l-1$, and sbp-1 mRNA in daf-2 and wild-type worms treated with $n s b p-1$-RNAi. qRT-PCR was performed to assess gene expression. ${ }^{*} p \leq 0.05$ versus control, $N S$ not significant. b Overexpression $(\mathrm{O} / \mathrm{E})$ of $n s b p-1$ extended lifespan. Mean lifespan of the wild type $17.2 \pm 0.1(n=96) ; n s b p-1(O / E) 21.6 \pm 0.5(n=107), p=0.0025$; $n s b p-1$ T203A $22.0 \pm 0.5(n=164), p<0.0001$; mean lifespan of daf-16 $13.3 \pm 0.2(n=108)$; daf-16; nsbp-1(O/E) $13.0 \pm 0.5$ $(n=133), p=0.63$. c Overexpression $(\mathrm{O} / \mathrm{E})$ of $n s b p-1$ increased resistance to oxidative stress. Worms $(n=30$ for each strain) were treated with paraquat, and then survival was assessed. In the

similar manner in mammals, including humans. NSBP-1 is a homolog of human NAP1, which is involved in such biological functions as histone chaperoning, nucleocytoplasmic shuttling, chromatin remodeling, enzyme inhibition, replication, transcription, silencing, and apoptosis [52]. This protein is essential in mammals since deletion leads to embryonic lethality or poor viability in adults [53, 54], similar to homozygous deletion of $n s b p-1$ in C. elegans.

NSBP-1 has cholesterol-binding activity. It possesses binding affinity for cholesterol $\left(K_{\mathrm{d}}=87 \mathrm{nM}\right.$; Fig. 1) comparable to that of other known sterol-binding proteins

wild-type background, survival rate was $n s b p-1(+) 61 \%$; $n s b p$ $1(\mathrm{O} / \mathrm{E}) 78 \%, p<0.001$ at $20 \mathrm{~h}$. In the daf-16 background, $n s b p-1$ overexpression did not improve survival rate. $p \leq 0.001$ versus $n s b p$ $1(+), N S$ not significant. d-g Fat storage measured by Oil-Red $\mathrm{O}$ staining. Fat storage in both the wild type $(\mathbf{d}-\mathbf{f})$ and daf-16 $(\mathbf{g}-\mathbf{h})$ increased when $n s b p-1$ was overexpressed. A representative image from one of three independent experiments $(n=20)$ is shown. $\mathbf{i} n s b p$ $1(O / E)$ upregulated $\operatorname{sod}-3$, and this upregulation depended on DAF-16 activity. sod-3 expression determined by qRT-PCR. ${ }^{*} p \leq 0.05$ versus each control, $N S$ not significant

(e.g., PBR, $K_{\mathrm{d}}=10 \mathrm{nM} ; \mathrm{NPC} 2, K_{\mathrm{d}}=30-50 \mathrm{nM} ; \mathrm{NPC} 1$, $K_{\mathrm{d}}=100 \mathrm{nM}$ ) [55-57]. Interestingly, like NPC2, NSBP-1 lacks any known sterol-sensing domain. Because NPC2 binds sterols via a hydrophobic region within its $\beta$-sheet [57], we speculate that NSBP-1 may bind to cholesterol via a highly conserved hydrophobic region within the $\beta$-sheet [52].

Another important function of NSBP-1 is to regulate DAF-16 activity. Although NAP1 has been reported to bind other transcription factors [58, 59], we show differential binding of NSBP-1 to a FOXO factor in C. elegans. Unlike 
Fig. 7 Proposed model for the potential role of NSBP-1 in the insulin pathway. DAF-2 signals through the AGE-1 signaling cascade activate AKT- 1 by phosphorylation. These kinases in turn regulate DAF-16 and NSBP-1 negatively. In the absence of insulin signaling, NSBP-1 binds to DAF-16 in the nucleus to regulate insulin signaling-mediated dauer formation, fat storage, and oxidative stress

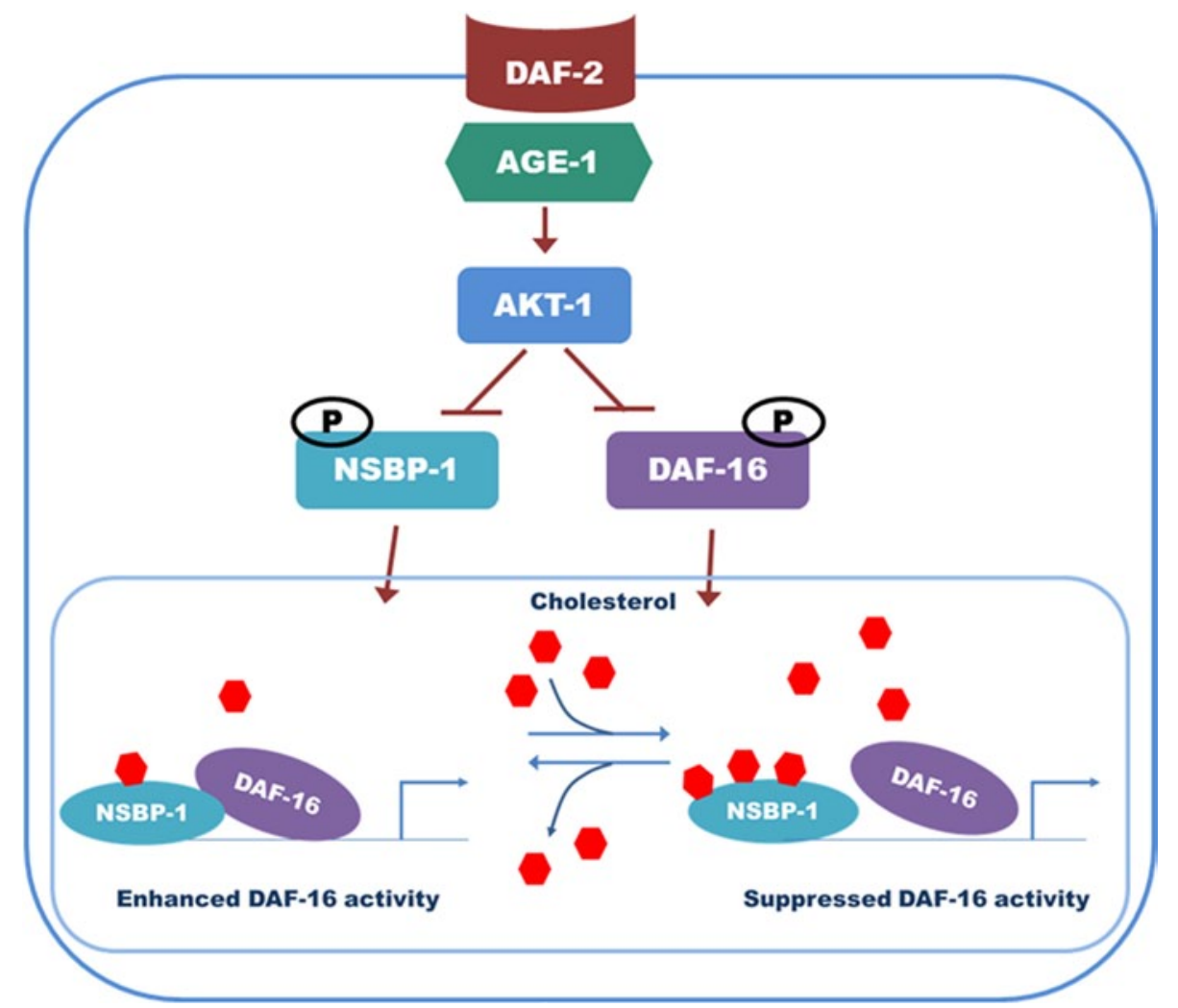

other nuclear factors (e.g., SMK-1, HCF-1, and BAR-1), NSBP-1 not only binds differentially to DAF-16 to control some of insulin signaling in a cholesterol-dependent manner, but also translocates to the nucleus depending on AKT. DAF-16 plays a major role in mediating changes in resource allocation in response to environmental changes such as heat stress and starvation [60]. DAF-16 translocates to the nucleus upon heat stress [60], whereas NSBP-1 does not seem to respond to this stress: there was no change in location of DAF-16 or NSBP-1 in response to changes in cholesterol level (data not shown). So we suggested that $n s b p-1$ does not exactly overlap with daf-16 function while cholesterol imbalance is different from stresses (e.g., heat stress).

Based on our results, we propose a model of how NSBP-1 mediates various cellular functions (Fig. 7). In the presence of active insulin signal, NSBP-1 and DAF-16 remain in the cytosol. In the absence of insulin signal, however, NSBP-1 translocates to the nucleus and interacts with DAF-16 to enhance its activity. This interaction increases dauer formation, oxidative stress resistance, and fat storage. However, under low- or high-cholesterol conditions, NSBP-1 can bind only weakly to DAF-16 and therefore DAF-16 activity is reduced (Figs. 5, 7). We interpret these data that the interaction between NSBP-1 and DAF-16 is dependent on cholesterol concentration and that interaction with DAF-16 is critical for NSBP-1-mediated regulation of the insulin signaling pathway. Studies showed that hyperactivation of
FOXO transcription factors causes insulin-resistant diabetes in mammals $[61,62]$. To prevent FOXO hyperactivation, a feedback control mechanism modulates the insulin signaling pathway [63]. Our finding that NSBP-1 as a cholesterolbinding protein regulates DAF-16 activity suggests that to prevent FOXO hyperactivation in worms, high cholesterol may suppress DAF-16 activity by controlling its binding to NSBP-1. In fact, DAF-16 hyperactivation could also be harmful to worms [64]. Thus, it is reasonable to propose that NSBP-1 acts as a cholesterol-dependent modulator of DAF16 function, which can lead to fine-tuning of aging, dauer formation, fat storage, and stress resistance in $C$. elegans. In mammals, binding of $\mathrm{CBP} / \mathrm{p} 300$ to FOXO factors is essential for FOXO-mediated transcription [65], and NAP1-p300 complexes promote transcriptional activation [66]. Our results suggest that NAP1-FoxO complexes could also play an important role in mammals, as NSBP-1-DAF-16 does in C. elegans.

In conclusion, we report the discovery of NSBP-1 as a novel cholesterol-binding protein that mediates many cellular processes by differentially interacting with DAF-16 in response to cholesterol concentration. Insulin signalingdependent nucleocytoplasmic translocation of NSBP-1 was found to be modulated in a cholesterol concentrationdependent manner. This finding expands our understanding of insulin resistance, as well as other metabolic disease mechanisms in mammals, suggesting screens for new 
therapeutic drugs for type II diabetes or treatment strategies for other metabolic syndromes in humans [67].

Acknowledgments This work was supported by National Research Foundation of Korea grant 2011-0028112 (to Y.-K. P.), World Class University Program grant R31-2008-000-10086-0 funded by the Korean government (Ministry of Education, Science and Technology), and National Project for Personalized Genomic Medicine grant A111218-11-CP01 (to Y.-K. P.). We also thank the National BioResource Project (NBRP) of Japan for providing the $n s b p-1(t m 1278)$ strain.

\section{References}

1. Peet DJ, Janowski BA, Mangelsdorf DJ (1998) The LXRs: a new class of oxysterol receptors. Curr Opin Genet Dev 8(5):571-575

2. Goldstein JL, DeBose-Boyd RA, Brown MS (2006) Protein sensors for membrane sterols. Cell 124(1):35-46. doi:10.1016/j. cell.2005.12.022

3. Cheruku SR, Xu Z, Dutia R, Lobel P, Storch J (2006) Mechanism of cholesterol transfer from the Niemann-Pick type $\mathrm{C} 2$ protein to model membranes supports a role in lysosomal cholesterol transport. J Biol Chem 281(42):31594-31604. doi:10.1074/jbc. M602765200

4. Borresen AL, Berg K (1981) Serum reserve cholesterol binding capacity (SRCBC): the relative importance of different lipoprotein classes. Artery 9(2):96-119

5. Scheel J, Srinivasan J, Honnert U, Henske A, Kurzchalia TV (1999) Involvement of caveolin-1 in meiotic cell-cycle progression in Caenorhabditis elegans. Nat Cell Biol 1(2):127-129. doi: $10.1038 / 10100$

6. Matyash V, Entchev EV, Mende F, Wilsch-Brauninger M, Thiele C, Schmidt AW, Knolker HJ, Ward S, Kurzchalia TV (2004) Sterol-derived hormone(s) controls entry into diapause in Caenorhabditis elegans by consecutive activation of DAF-12 and DAF16. PLoS Biol 2(10):e280. doi:10.1371/journal.pbio.0020280

7. Shim YH, Chun JH, Lee EY, Paik YK (2002) Role of cholesterol in germ-line development of Caenorhabditis elegans. Mol Reprod Dev 61(3):358-366. doi:10.1002/mrd.10099

8. Yochem J, Tuck S, Greenwald I, Han M (1999) A gp330/megalinrelated protein is required in the major epidermis of Caenorhabditis elegans for completion of molting. Development 126(3):597-606

9. Paik YK, Jeong SK, Lee EY, Jeong PY, Shim YH (2006) C. elegans: an invaluable model organism for the proteomics studies of the cholesterol-mediated signaling pathway. Expert Rev Proteomics 3(4):439-453. doi:10.1586/14789450.3.4.439

10. Cheong MC, Na K, Kim H, Jeong SK, Joo HJ, Chitwood DJ, Paik YK (2011) A potential biochemical mechanism underlying the influence of sterol deprivation stress on Caenorhabditis elegans longevity. J Biol Chem 286(9):7248-7256. doi:10.1074/jbc. M110.189183

11. Gerisch B, Weitzel C, Kober-Eisermann C, Rottiers V, Antebi A (2001) A hormonal signaling pathway influencing C. elegans metabolism, reproductive development, and life span. Devel Cell 1(6):841-851

12. Saltiel AR, Kahn CR (2001) Insulin signalling and the regulation of glucose and lipid metabolism. Nature 414(6865):799-806. doi: $10.1038 / 414799$ a

13. Accili D (2004) Lilly lecture 2003: the struggle for mastery in insulin action: from triumvirate to republic. Diabetes 53 (7):16331642. pii:53/7/1633
14. Cohen E, Dillin A (2008) The insulin paradox: aging, proteotoxicity and neurodegeneration. Nat Rev Neurosci 9(10):759-767. doi: $10.1038 / \mathrm{nrn} 2474$

15. Antebi A (2007) Genetics of aging in Caenorhabditis elegans. PLoS Genet 3(9):1565-1571. doi:10.1371/journal.pgen.0030129

16. Kenyon C (2005) The plasticity of aging: insights from long-lived mutants. Cell 120(4):449-460. doi:10.1016/j.cell.2005.02.002

17. Kimura KD, Tissenbaum HA, Liu Y, Ruvkun G (1997) daf-2, an insulin receptor-like gene that regulates longevity and diapause in Caenorhabditis elegans. Science 277(5328):942-946

18. Morris JZ, Tissenbaum HA, Ruvkun G (1996) A phosphatidylinositol-3-OH kinase family member regulating longevity and diapause in Caenorhabditis elegans. Nature 382(6591):536-539. doi:10.1038/382536a0

19. Hertweck M, Gobel C, Baumeister R (2004) C. elegans SGK-1 is the critical component in the Akt/PKB kinase complex to control stress response and life span. Dev Cell 6 (4):577-588. pii:S1534580704000954

20. Paradis S, Ailion M, Toker A, Thomas JH, Ruvkun G (1999) A PDK1 homolog is necessary and sufficient to transduce AGE-1 PI3 kinase signals that regulate diapause in Caenorhabditis elegans. Genes Dev 13(11):1438-1452

21. Paradis S, Ruvkun G (1998) Caenorhabditis elegans Akt/PKB transduces insulin receptor-like signals from AGE-1 PI3 kinase to the DAF-16 transcription factor. Genes Dev 12(16):2488-2498

22. Lin K, Dorman JB, Rodan A, Kenyon C (1997) daf-16: an HNF3/forkhead family member that can function to double the lifespan of Caenorhabditis elegans. Science 278(5341):1319-1322

23. Ogg S, Paradis S, Gottlieb S, Patterson GI, Lee L, Tissenbaum HA, Ruvkun G (1997) The Fork head transcription factor DAF16 transduces insulin-like metabolic and longevity signals in $C$. elegans. Nature 389(6654):994-999. doi:10.1038/40194

24. Gems D, Sutton AJ, Sundermeyer ML, Albert PS, King KV, Edgley ML, Larsen PL, Riddle DL (1998) Two pleiotropic classes of daf-2 mutation affect larval arrest, adult behavior, reproduction and longevity in Caenorhabditis elegans. Genetics 150(1):129-155

25. Calnan DR, Brunet A (2008) The FoxO code. Oncogene 27(16):2276-2288. doi:10.1038/onc.2008.21

26. Brenner S (1974) The genetics of Caenorhabditis elegans. Genetics 77(1):71-94

27. Cuatrecasas P, Wilchek M, Anfinsen CB (1968) Selective enzyme purification by affinity chromatography. Proc Natl Acad Sci USA 61(2):636-643

28. Cuatrecasas $P$ (1970) Protein purification by affinity chromatography. Derivatizations of agarose and polyacrylamide beads. J Biol Chem 245(12):3059-3065

29. Shaltiel S, Er-El Z (1973) Hydrophobic chromatography: use for purification of glycogen synthetase. Proc Natl Acad Sci USA 70(3):778-781

30. Suhara K, Gomi T, Sato H, Itagaki E, Takemori S, Katagiri M (1978) Purification and immunochemical characterization of the two adrenal cortex mitochondrial cytochrome P-450-proteins. Arch Biochem Biophys 190(1):290-299. doi:0003-9861(78)90278-3

31. Gibson GG, Schenkman JB (1978) Purification and properties of cytochrome P-450 obtained from liver microsomes of untreated rats by lauric acid affinity chromatography. J Biol Chem 253(17):5957-5963

32. Lee HJ, Kwon MS, Lee EY, Cho SY, Paik YK (2008) Establishment of a PF2D-MS/MS platform for rapid profiling and semiquantitative analysis of membrane protein biomarkers. Proteomics 8(11):2168-2177. doi:10.1002/pmic.200701022

33. Radhakrishnan A, Sun LP, Kwon HJ, Brown MS, Goldstein JL (2004) Direct binding of cholesterol to the purified membrane region of SCAP: mechanism for a sterol-sensing domain. Mol Cell 15(2):259-268. doi:10.1016/j.molcel.2004.06.019 
34. Dong MQ, Venable JD, Au N, Xu T, Park SK, Cociorva D, Johnson JR, Dillin A, Yates JR, 3rd (2007) Quantitative mass spectrometry identifies insulin signaling targets in C. elegans. Science 317 (5838):660-663. doi:10.1126/science.1139952

35. Rourke EJ, Soukas AA, Carr CE, Ruvkun G (2009) C. elegans major fats are stored in vesicles distinct from lysosome-related organelles. Cell Metabol 10(5):430-435. doi:10.1016/j.cmet.2009.10.002

36. Ristow M, Pfister MF, Yee AJ, Schubert M, Michael L, Zhang CY, Ueki K, Michael MD 2nd, Lowell BB, Kahn CR (2000) Frataxin activates mitochondrial energy conversion and oxidative phosphorylation. Proc Natl Acad Sci USA 97(22):12239-12243. doi:10.1 073/pnas.220403797

37. Matyash V, Geier C, Henske A, Mukherjee S, Hirsh D, Thiele C, Grant B, Maxfield FR, Kurzchalia TV (2001) Distribution and transport of cholesterol in Caenorhabditis elegans. Mol Biol Cell 12(6): $1725-1736$

38. Park YJ, Luger K (2006) Structure and function of nucleosome assembly proteins. Biochem Cell Biol 84(4):549-558. doi: $10.1139 / 006-088$

39. Wyles JP, Perry RJ, Ridgway ND (2007) Characterization of the sterol-binding domain of oxysterol-binding protein (OSBP)related protein 4 reveals a novel role in vimentin organization. Exp Cell Res 313(7):1426-1437. doi:10.1016/j.yexcr.2007.01.018

40. Fielenbach N, Antebi A (2008) C. elegans dauer formation and the molecular basis of plasticity. Genes Dev 22(16):2149-2165. doi:10.1101/gad.1701508

41. Lithgow GJ, Walker GA (2002) Stress resistance as a determinate of C. elegans lifespan. Mech Ageing Dev 123 (7):765-771.pii:S0047637401004225

42. Lin K, Hsin H, Libina N, Kenyon C (2001) Regulation of the Caenorhabditis elegans longevity protein DAF-16 by insulin/IGF-1 and germline signaling. Nat Genet 28(2):139-145. doi:10.1038/88850

43. Tullet JM, Hertweck M, An JH, Baker J, Hwang JY, Liu S, Oliveira RP, Baumeister R, Blackwell TK (2008) Direct inhibition of the longevity-promoting factor $\mathrm{SKN}-1$ by insulin-like signaling in C. elegans. Cell 132 (6):1025-1038. doi:10.1016/j.cell.2008.01.030

44. Calvert ME, Keck KM, Ptak C, Shabanowitz J, Hunt DF, Pemberton LF (2008) Phosphorylation by casein kinase 2 regulates Nap1 localization and function. Mol Cell Biol 28(4):1313-1325. doi:10.1128/MCB.01035-07

45. Phillips JE, Gersbach CA, Wojtowicz AM, Garcia AJ (2006) Glucocorticoid-induced osteogenesis is negatively regulated by Runx2/Cbfa1 serine phosphorylation. J Cell Sci 119(Pt 3):581591. doi: $10.1242 /$ jcs. 02758

46. Brown MS, Goldstein JL (1997) The SREBP pathway: regulation of cholesterol metabolism by proteolysis of a membrane-bound transcription factor. Cell 89(3):331-340

47. Kamagate A, Qu S, Perdomo G, Su D, Kim DH, Slusher S, Meseck M, Dong HH (2008) FoxO1 mediates insulin-dependent regulation of hepatic VLDL production in mice. J Clin Invest 118(6):2347-2364. doi:10.1172/JCI32914

48. Choi BK, Chitwood DJ, Paik YK (2003) Proteomic changes during disturbance of cholesterol metabolism by azacoprostane treatment in Caenorhabditis elegans. Mol Cell Proteomics 2(10):10861095. doi:10.1074/mcp.M300036-MCP200M300036-MCP200

49. Merris M, Kraeft J, Tint GS, Lenard J (2004) Long-term effects of sterol depletion in C. elegans: sterol content of synchronized wild-type and mutant populations. J Lipid Res 45(11):2044-2051. doi:10.1194/jlr.M400100-JLR200M400100-JLR200

50. Lee EY, Shim YH, Chitwood DJ, Hwang SB, Lee J, Paik YK (2005) Cholesterol-producing transgenic Caenorhabditis elegans lives longer due to newly acquired enhanced stress resistance. Biochem Biophys Res Commun 328(4):929-936. doi:10.1016/j.bbrc.2005.01.050
51. Li J, Brown G, Ailion M, Lee S, Thomas JH (2004) NCR-1 and NCR-2, the C. elegans homologs of the human Niemann-Pick type $\mathrm{C} 1$ disease protein, function upstream of DAF-9 in the dauer formation pathways. Development 131(22):5741-5752. doi:10.1242/dev.01408

52. Park YJ, Luger K (2006) The structure of nucleosome assembly protein 1. Proc Nat Acad Sci USA 103(5):1248-1253. doi:10.107 3/pnas.0508002103

53. Lankenau S, Barnickel T, Marhold J, Lyko F, Mechler BM, Lankenau DH (2003) Knockout targeting of the Drosophila nap1 gene and examination of DNA repair tracts in the recombination products. Genetics 163(2):611-623

54. Rogner UC, Spyropoulos DD, Le Novere N, Changeux JP, Avner $\mathrm{P}$ (2000) Control of neurulation by the nucleosome assembly protein-1-like 2. Nat Genet 25(4):431-435. doi:10.1038/78124

55. Infante RE, Abi-Mosleh L, Radhakrishnan A, Dale JD, Brown MS, Goldstein JL (2008) Purified NPC1 protein. I. Binding of cholesterol and oxysterols to a 1278 -amino acid membrane protein. J Biol Chem 283(2):1052-1063. doi:10.1074/jbc.M707943200

56. Jamin N, Neumann JM, Ostuni MA, Vu TK, Yao ZX, Murail S, Robert JC, Giatzakis C, Papadopoulos V, Lacapere JJ (2005) Characterization of the cholesterol recognition amino acid consensus sequence of the peripheral-type benzodiazepine receptor. Mol Endocrinol 19(3):588-594. doi:10.1210/me.2004-0308

57. Ko DC, Binkley J, Sidow A, Scott MP (2003) The integrity of a cholesterol-binding pocket in Niemann-Pick C2 protein is necessary to control lysosome cholesterol levels. Proc Natl Acad Sci USA 100(5):2518-2525. doi:10.1073/pnas.05300271000530027100

58. Eckey M, Hong W, Papaioannou M, Baniahmad A (2007) The nucleosome assembly activity of NAP1 is enhanced by Alien. Mol Cell Biol 27(10):3557-3568. doi:10.1128/MCB.01106-06

59. Shikama N, Chan HM, Krstic-Demonacos M, Smith L, Lee CW, Cairns W, La Thangue NB (2000) Functional interaction between nucleosome assembly proteins and p300/CREB-binding protein family coactivators. Mol Cell Biol 20(23):8933-8943

60. Henderson ST, Johnson TE (2001) daf-16 integrates developmental and environmental inputs to mediate aging in the nematode Caenorhabditis elegans. Curr Biol 11 (24):1975-1980. pii: S0960-9822(01)00594-2

61. Biddinger SB, Haas JT, Yu BB, Bezy O, Jing E, Zhang W, Unterman TG, Carey MC, Kahn CR (2008) Hepatic insulin resistance directly promotes formation of cholesterol gallstones. Nat Med 14(7):778-782. doi:10.1038/nm1785

62. Matsumoto M, Han S, Kitamura T, Accili D (2006) Dual role of transcription factor FoxO1 in controlling hepatic insulin sensitivity and lipid metabolism. J Clin Invest 116(9):2464-2472. doi:10.1172/JCI27047

63. Puig O, Tjian R (2005) Transcriptional feedback control of insulin receptor by dFOXO/FOXO1. Genes Dev 19(20):2435-2446. doi:10.1101/gad.1340505

64. Libina N, Berman JR, Kenyon C (2003) Tissue-specific activities of C. elegans DAF-16 in the regulation of lifespan. Cell 115 (4):489-502. pii:S0092867403008894

65. van der Heide LP, Smidt MP (2005) Regulation of FoxO activity by $\mathrm{CBP} / \mathrm{p} 300-$ mediated acetylation. Trends Biochem Sci 30(2):81-86. doi:10.1016/j.tibs.2004.12.002

66. Asahara H, Tartare-Deckert S, Nakagawa T, Ikehara T, Hirose F, Hunter T, Ito T, Montminy M (2002) Dual roles of p300 in chromatin assembly and transcriptional activation in cooperation with nucleosome assembly protein 1 in vitro. Mol Cell Biol 22(9):2974-2983

67. Mooradian AD (2009) Dyslipidemia in type 2 diabetes mellitus. Nat Clin Pract Endocrinol Metab 5(3):150-159. doi:10.1038/ncpe ndmet1066 